\title{
Educación Patrimonial una Pedagogía desde Museos Populares y/o Comunitarios
}

\author{
Buitrón Chávez, Santiago \\ s.buitron@awasqa.com \\ Coordinador Regional de la Red de Gestión Cultural Comunitaria \\ AWASQA
}

\section{RESUMEN}

El sincretismo cultural, genera estructuras de evolución social, determinando fases de discernimiento comportamental que pueden ser evidenciadas a través de fenómenos o eventos que desencadenan procesos comunitarios. La conciliación de este coexistir y del compartir convivencias, tiene un fuerte potencial de generación de creatividades nuevas, las mismas que pueden irse interconectando y moldeando en dinámicas con identidad propia, cada vez más enriquecidas por el aporte espontáneo de las sensibilidades y las percepciones que se producen dentro de comunidades, sean urbanas o rurales.

Esta mezcla natural de contenidos creativos, a través de la convivencia social, determina la aparición de legados, que se los pueden catalogar desde discernimientos íntimos y locales; de tal forma que lo creado, como esencia vital de una manifestación comunitaria, sea además puesto en escena para la consideración, divulgación y promoción de los patrimonios.

El presente documento constituye un breve resumen teórico de una investigación doctoral en la cual se logra determinar un lineamiento pedagógico y comunicativo a partir de espacios museísticos populares, dentro de los cuales se evidencia un encuentro significativo de lo comunitario, como generador de legados, que pueden ser catalogados como patrimonios.

Palabras claves: Educación, Patrimonio, Cultura, Comunitario, Museo, Popular. 


\title{
Heritage Education a Pedagogy from Popular and / or Community
}

\section{Museums}

\begin{abstract}
Cultural syncretism generates structures of social evolution, determining phases of behavioural discernment that can be evidenced through phenomena or events that trigger community processes. The reconciliation of this coexistence and the sharing of coexistences, has a strong potential to generate new creatives, the same that can be interconnected and molded into dynamics with their own identity, increasingly enriched by the spontaneous contribution of sensitivities and perceptions that occur within communities, whether urban or rural.

This natural mix of creative content, through social coexistence, determines the emergence of legacies, which can be catalogued from intimate and local discernments; in such a way that what is created, as the vital essence of a Community demonstration, is also staged for the consideration, dissemination and promotion of heritage.

This document is a brief theoretical summary of a doctoral research in which it is possible to determine a pedagogical and communicative guideline from popular museum spaces, within which a significant encounter of the community is evident, as a generator of legacies, which can be classified as heritage.
\end{abstract}

Keywords: Education, Heritage, Culture, Community, Museum, Popular.

Artículo recibido: 15 abril 2021

Aceptado para publicación: 19 abril 2021

Correspondencia: s.buitron@awasqa.com

Conflictos de Interés: Ninguna que declarar 


\section{Epistemología}

Bajo el delimitante polisémico del vocablo cultura, y la relación de esta con el término patrimonio, se tiene un lineamiento bifocal cultura-patrimonio que estructura un paradigma de generación de legados a través del interaccionar social de las comunidades, ya sea de forma intrínseca, así como también de una manera externa a partir del encuentro con otros círculos afines (o no) de conglomerados humanos.

Se toma así, como un eje central paradigmático para profundizar en el tema que se trata; y teniendo en cuenta que "un paradigma es la concepción del objeto de estudio de una ciencia acompañada de un conjunto de teorías básicas sobre aspectos particulares de ese objeto. Ese contenido define los problemas que deben investigarse, la metodología por emplear y la forma de explicar los resultados de la investigación”. (Briones, 2002), de ahí que, en este caso, el concepto patrimonio acompaña a la cultura para delimitarla a la convivencia humana y la generación de productos sociales (tangibles o intangibles).

Y es precisamente, el proceso de darle un campo de acción específico para la temática, que se hace la relación cultura-patrimonio como un solo momento de reflexión sobre lo creado socialmente; por lo que "desde uno de los puntos de vista de la epistemología, la preocupación principal del filósofo alemán Heinrich Rickert (1863 - 1936) está dirigida a encontrar la diferencia básica entre las ciencias naturales y las que él denomina ciencias de la cultura..." (Briones, 2002); y así también, desde esta dualidad se hace una alusión a los legados estéticos haciendo mención al carácter científico de observar tanto al proceso de construcción como a los productos generados.

\section{Al respecto:}

Ahora podemos preguntarnos cuál es el método propio de las ciencias culturales. La respuesta de Rickert es la siguiente: es el método individualizador o idiográfico. Las ciencias culturales o históricas, en sentido amplio, no buscan generalizaciones, no pretenden formular leyes: su tarea consiste en la búsqueda de singularidades, de los aspectos particulares que individualizan un determinado fenómeno. (Briones, 2002)

Se reitera el carácter de la especificidad, precisamente para no complicarse en la ambigüedad de la amplitud de entendimientos; por lo cual cabe aquella referencia en la que el estudio de la cultura también es un trabajo desde lo histórico y lo singular; de tal forma que ajustando un poco más a la línea de investigación se la puede estar ubicando dentro de la etnometodología, la misma que "aparece sistematizada por primera vez en la 
obra de Garfinkel, en 1967 (...) como el estudio de los conocimientos del sentido común y de los métodos y procedimientos que las personas corrientes utilizan para darle sentido a las situaciones en la cuales se encuentran y hallan el camino que deben seguir en ellas y, en consecuencia, actúan. (Briones, 2002)

Destaca un texto de Freire (2005) que sirve de punto de partida para el entendimiento del presente estudio:

La objetividad dicotomizada de la subjetividad, la negación de ésta en el análisis de la realidad o en la acción sobre ella, es objetivismo. De la misma forma, la negación de la objetividad, en el análisis como en la acción, por conducir al subjetivismo que se extiende en posiciones solipsistas, niega la acción misma, al negar la realidad objetiva, desde el momento en que ésta pasa a ser creación de la conciencia. Ni objetivismo, ni subjetivismo o psicologismo, sino subjetividad y objetividad en permanente dialecticidad.

El patrimonio, como producto de la cultura, y legado de las sociedades para generaciones posteriores, se convierte en un objeto de tratamiento estético que obtiene un valor (económico, emocional, afectivo, simbólico social) por el cual se sustenta la afirmación del constante proceso de producción y re-creación que se halla implícito en toda convivencia humana.

El papel de la cultura en el desarrollo humano es insustituible. La cultura es el objetivo esencial de una adecuada concepción del desarrollo, es parte integral de dicho proceso y es un instrumento activo de la conducción del desarrollo social. (Gutiérrez, 2010). Los legados son parte de la evidencia de la vitalidad de una sociedad.

Para el caso de lo que se estudia en el tema presente, resulta válido aclarar el término desarrollo en términos de evolución social y no necesariamente en lo económico, aunque sí puede alcanzar lo tecnológico. (Gutiérrez, 2010) hace un acercamiento al respecto: "En tanto contenido del desarrollo social, la cultura se expresa como la interrelación dialéctica de los aspectos materiales y espirituales de la vida del hombre; como esfera superestructural, interactúa con la base económica de la sociedad y se concreta en el desarrollo de la cultura material y de la cultura espiritual".

\section{Educación Patrimonial}

A partir del mes de noviembre de 1972, una vez finalizada la Convención sobre la Protección del Patrimonio Mundial, Cultural y Natural de la UNESCO, se establecen de manera formal, definiciones y conceptos sobre términos que resultaban de amplio uso 
dentro del campo del patrimonio a nivel global, en un intento oportuno de homologar las acepciones sobre el tema y así estructurar de manera clara el documento del convenio, el mismo que serviría de base para la generación de políticas de protección, mantenimiento y gestión de espacios, sitios, productos y legados patrimoniales, en los diferentes territorios del mundo.

Dentro de los aspectos más importantes de la convención se determina que urge alentar a las naciones a garantizar la protección de los patrimonios a través de estrategias de comunicación, educación, investigación, formación y sensibilización del público; surge así desde aquella época el término: educación patrimonial.

De conformidad con la (UNESCO, 2014), se tiene que:

La noción de patrimonio es importante para la cultura y el desarrollo, en cuanto constituye el "capital cultural" de las sociedades contemporáneas. Contribuye a la revalorización continua de las culturas y de las identidades, y es un vehículo importante para la transmisión de experiencias, aptitudes y conocimientos entre las generaciones. Además, es fuente de inspiración para la creatividad y la innovación, que generan los productos, culturales, contemporáneos, y futuros.

La revalorización continua, la del capital cultural, estimula la estructuración de un currículo especializado en patrimonio, como un reflejo del permanente evolucionar de los comportamientos sociales y de sus imaginarios frente al accionar humano local, regional y global; procesos de convivencia social que van generando nuevos legados, los mismos que, tienen el potencial de ir definiendo espacios museísticos como territorios de estudio, investigación y transferencias de conocimiento e identidades culturales.

La propia definición del potencial del patrimonio como ente pedagógico sugiere el fortalecimiento de las condiciones socio-económicas de su área de influencia favoreciendo mejoras al estilo de vida de sus comunidades, ya que, al estar formado y guiado por la contextualidad de sus propios legados, la identificación y el sentido de apropiación de lo suyo será vigorizado desde la lectura de lo que ha sido y lo que puede llegar a ser.

\section{Sincretismo Cultural}

Los eventos de conciliación, desde el encuentro de culturas, han determinado un mestizaje global que dentro de un sistema mundo, identifican distinciones genéricas de comunidades que, resultan en apropiaciones de contextos para la asimilación y/o 
transformación social. Las culturas populares son un reflejo de lo afirmado, como parte de lo que ha permanecido invisibilizado, pero que quiere protagonizar una cierta reivindicación ante momentos de opresión dentro de procesos culturales relativamente jerarquizados.

Las culturas populares (más que la cultura popular) se configuran por un proceso de apropiación desigual de los bienes económicos y culturales de una nación o etnia por parte de sus sectores subalternos y por la comprensión, reproducción y transformación, real y simbólica, de las condiciones generales y propias de trabajo y vida. (García N. , 1982). Es el pueblo conformado por sus círculos de pensamiento que fluyen entre individuos desde su unicidad hasta la conformación de la comunidad creadora como tal, que crea desde su propia cosmovisión.

Freire (2005) profundiza y aclara lo que se argumenta en el párrafo anterior:

El círculo de cultura, en el método Paulo Freire, revive la vida en profundidad crítica. La conciencia emerge del mundo vivido, lo objetiva, lo problematiza, lo comprende como proyecto humano. En diálogo circular, intersubjetivándose más y más, va asumiendo críticamente el dinamismo de su subjetividad creadora. Todos juntos, en círculo, y en colaboración, reelaboran el mundo, y al reconstruirlo, perciben que, aunque construido también por ellos, ese mundo no es verdaderamente de ellos y para ellos. Humanizado por ellos, ese mundo los humaniza. Las manos que lo hacen no son las que lo dominan. Destinado a liberarlos como sujetos, los esclaviza como objetos.

Aquella trascendencia del ser hacia la inmortalización de su existencia por medio de sus legados, le da un carácter místico a la convivencia de las culturas, permitiendo que surjan apropiaciones simbólicas que detallan el pensamiento común, la vida y la prospectiva de la comunidad, de forma relativa hacia el desarrollo social desde lo cultural. "Durante bastante tiempo se creyó que el relativismo cultural (...) si bien ayuda a superar el etnocentrismo, deja abiertos problemas básicos en una teoría de la cultura: la construcción de un conocimiento de validez universal y de criterios que sirvan para pensar y resolver los conflictos y desigualdades interculturales”. (García N. , 1982); argumento que aporta a identificar que no hay culturas superiores ni inferiores, y que esto ayuda a la conciliación enunciada anteriormente e incluso fortalece el devenir cultural para la convivencia creativa y productiva. 
Por lo tanto, al dejar de lado la distinción entre cultura superior e inferior, y dando prioridad a una participación activa o pasiva en la convivencia cultural, se tiene la seguridad de que: “el análisis de una cultura no puede centrarse en los objetos o bienes culturales; debe ocuparse del proceso de producción y circulación social de los objetos y de los significados que diferentes receptores le atribuyen". (García N. , Las culturas populares en el capitalismo, 1982), y para este caso: los legados estéticos del convivir.

"La existencia, en tanto humana, no puede ser muda, silenciosa, ni tampoco nutrirse de falsas palabras sino de palabras verdaderas con las cuales los hombres transforman al mundo. Existir humanamente, es "pronunciar" el mundo, es transformarlo". (Freire, 2005), estos pronunciamientos generan conceptualizaciones que desarrollan continuas réplicas y permanentes aportes conceptuales para la re-creación social.

En síntesis: las culturas populares son el resultado de una apropiación desigual del capital cultural, una elaboración propia de sus condiciones de vida y una interacción conflictiva con los sectores hegemónicos. Al comprenderlas de este modo, nos alejamos de las dos posiciones que han predominado en su estudio: las interpretaciones inmanentes formuladas en Europa por el populismo romántico y en América Latina por el nacionalismo y el indigenismo conservadores; y, por otra parte, del positivismo que, preocupado por el rigor científico, olvidó el sentido político de la producción simbólica del pueblo. (García N. , 1982)

Y así, desde lo popular, haciendo alusión al pueblo, lo sincrético conduce una forma óptima de convivencia para la productividad; y en el momento en el que se sistematizan los productos, se identifican los legados que luego se los concibe como patrimonios. "El campo cultural puede ser todavía un laboratorio. (...) Frente a la "eficiencia" productivista, reivindica lo lúdico; ante la obsesión lucrativa, la libertad de retrabajar las herencias sin réditos que permanecen en la memoria, las experiencias no capitalizables que pueden librarnos de la monotonía y la inercia. (García N. , 1990)

\section{Convivencia social}

Se reitera que la convivencia, como evidencia de lo sincrético social, es un germen de información que se estructura en pensamiento y que se ejecuta como conocimiento; "es decir que ningún grupo "tiene" una cultura sólo por sí mismo: la cultura es el nimbo que percibe un grupo cuando entra en contacto con otro y lo observa". (Jameson, 2008). Entonces, el convivir social se manifiesta a través de la fusión de disciplinas que, implícita 
o explícitamente se encuentran presentes en las rutinas vitales de los individuos en una comunidad.

Gutiérrez (2010) hace referencia a las "organizaciones", las mismas que "son sistemas complejos que mantienen permanentes intercambios con el entorno, desarrollando procesos de autorregulación y retroalimentación. Procesos interactivos que se producen también hacia su interior, donde se gestiona el desarrollo de la organización y donde gran parte de los intercambios son simbólicos, o sea, comunicacionales", con esta afirmación se establece además el hecho de que la convivencia social se mantiene a través de procesos organizados.

La convivencia se destaca además por los intercambios:

El intercambio con otros pueblos a través del comercio, la migración o los medios de comunicación hacen que entremos en contacto y nos identifiquemos con ciertos elementos culturales producidos fuera de nuestro territorio y los adoptemos. Es por ello que muchas manifestaciones culturales mantienen su sentido y función originales a través del tiempo, son parte de la memoria histórica como parte de la identidad y otras dejan de formar parte del acervo de un pueblo. (Mejía, 2007)

Se reitera el acto de la convivencia como una acción de aceptación de las culturas para su enriquecimiento mutuo desde el aporte de sus costumbres y rutinas vitales; anteponiéndose al capitalismo global en relación a un multiculturalismo, "esa actitud que -desde una suene de posición global vacía- trata a cada cultura local como el colonizador trata al pueblo colonizado: como "nativos", cuya mayoría debe ser estudiada y "respetada" cuidadosamente. (Jameson, 2008); y es precisamente esto lo que se debe evitar dentro de un proceso de convivencia para la generación de legados.

\section{Estéticas y legados}

En referencia al legado estético del convivir, puede tomarse como cierto el hecho de todo lo antiguo tiene su belleza, desde lo introyectivo que, corresponde a la evocación de lo vivido, hasta lo más explícito que, deviene del recuerdo hacia la concreción de las decisiones que aluden a la continuidad de una cultura que, basa sus prospectivas a partir del conocimiento de sus orígenes. De manera que el discurso estético ha dejado de ser la representación del discurso creador apara convertirse en un recurso complementario destinado a "garantizar" la verosimilitud de la experiencia artística en el momento de consumo. (García N. , 1990) 
Lo estético tiene su paralelismo en una forma de belleza desde la cual se puede manifestar una cultura en relación con otra, haciendo verídica la relatividad de lo estético en función del observador y de su contexto, y en ello la época contemporánea, con la cantidad basta de estímulos sensoriales con los que cuenta para alimentar las culturas, es un detonador de nuevas creatividades. Al respecto "pensamos que el impulso innovador y expansivo de la modernidad está tocando su techo, pero tal vez esto permite pensar en otros modos de innovación que no sean la evolución incesante hacia lo desconocido" (García N. , 1990).

En otras palabras, en vez de lamentar la desintegración de la vida comunitaria debido al impacto de las nuevas tecnologías, resulta mucho más interesante analizar cómo el progreso tecnológico en sí mismo da origen a nuevas comunidades que gradualmente se "naturalizan", como el caso de las comunidades virtuales. (Jameson, 2008), y la estética se mantiene siempre latente, y en permanente estado de evolución y re-creación; así como también generación de legados, haciendo de la virtualidad una cultura post-urbana. En relación a esto "la 'cultura urbana' es reestructurada al ceder al protagonismo del espacio público a las tecnologías electrónicas. Al "pasar" casi todo en la ciudad gracias a que los medios lo dicen, y al parecer que ocurre como los medios quieren, se acentúa la mediatización social, el peso de las escenificaciones, las acciones políticas se constituyen en tanto imagen de lo político". (García N. , 1990)

\section{Legados y patrimonios}

Una concepción general de patrimonio se tiene de la Organización de las Naciones Unidas para la Educación, Ciencia y Cultura, como elemento de referencia a nivel internacional, e institución desde la cual se emiten lineamientos acerca del tema. Es así que:

La gama de lo que se considera patrimonio se ha extendido mucho en el último medio siglo. Los bienes del patrimonio solían ser monumentos individuales o edificios tales como lugares de culto o fortificaciones, y con frecuencia se les veía como hitos singulares sin relación especial con el paisaje circundante. Hoy en día existe un mayor reconocimiento de que la interacción del medio ambiente con la humanidad lo ha afectado en su totalidad y que, en consecuencia, reúne las condiciones para ser reconocido como patrimonio. Cada vez es más necesario pronunciarse acerca de lo que es significativo y lo que no lo es. (UNESCO, Gestión del Patrimonio Mundial Cultural, 2014) 
Acotando de más de que, de forma básica se tiene como patrimonio a legados tangibles e intangibles.

Esta sensorialidad de los legados permite ubicarlos dentro de prioridades aun discutibles: ¿es más importante un patrimonio tangible o un patrimonio intangible? La valoración de los mismos estará determinada por múltiples variables. "El proceso mediante el cual nos identificamos con ciertos valores, símbolos, relatos y formas de ser y que adoptamos como nuestro patrimonio, hace referencia a la identidad cultural que tiene como base una particular visión de la vida y del mundo". (Mejía, 2007); algunas de ellas explicadas en lo citado en este párrafo.

Un aporte de valoración, para la gestión, también se lo encuentra en la interpretación cultural:

Al mismo tiempo, hay que admitir que existe una serie de características de la interpretación cultural que hacen el desarrollo teórico mucho más difícil de lo que suele ser en otras disciplinas. La primera característica es la necesidad de que la teoría permanezca más cerca del terreno estudiado de lo que permanece en el caso de ciencias más capaces de entregarse a la abstracción imaginativa. (Geertz, 2003)

Es decir, una teorización metodológica permite también ubicar un legado en función de elementos de productividad que determinarán una utilidad, no solamente económica, sino también en base a la conservación de elementos vitales para la existencia de una comunidad. Esta importante valoración se la encuentra en (Mejía, 2007):

Las manifestaciones no materiales emanan de una cultura en forma de saberes (conocimientos y modos de hacer comunidades), celebraciones (rituales, festividades, prácticas de la vida social), formas de expresión (manifestaciones literarias, musicales, plásticas, escénicas, lúdicas, entre otras) y lugares (mercados, ferias, santuarios, plazas y demás espacios donde tienen lugar prácticas culturales).

En referencia a lo tangible e intangible del patrimonio como parte de un legado heredado en el transcurrir de las generaciones y que va alimentándose constantemente de nuevas manifestaciones sociales de las culturas que también se renuevan de forma continua.

\section{Gestión del patrimonio como recurso de productividad}

En cuanto a gestión del Patrimonio, volvemos a la UNESCO:

Se identifican nueve características básicas (o "mínimos comunes denominadores") que están presentes en todos los sistemas de gestión del patrimonio... A continuación, se 
agrupan las nueve características en tres elementos (marco jurídico, marco institucional y recursos), tres procesos (planificación, ejecución y monitoreo) y tres resultados (productos, resultados y mejoras). (UNESCO, Gestión del Patrimonio Mundial Cultural, 2014)

Razón por lo que el legado estético del convivir requiere de una gestión que alimente las posibilidades de creación y de generación de propuestas que sean la evidencia del ser de una comunidad.

Un elemento de motivación hacia la gestión óptima del patrimonio se lo encuentra en la modernidad ( $\sin$ la necesidad de profundizar en el término), entendiéndose como un proceso de mixtura global entre culturas, es así que, urge sistematizar las incontables posibilidades de modos o mecanismos de establecer políticas para sostener procesos de valoración de patrimonios. "Las emergencias provocadas por la diversidad de interacciones reconocibles generan procesos de auto-organización, y ésta se identifica, así como propiedad de la emergencia". (Gutiérrez, 2010); las comunidades tienen en su espíritu más profundo un sentido en el cual saben que deben mantener una línea de transferencia de conocimientos a través de sus legados.

Por lo que, en correspondencia con la modernidad:

Las búsquedas más radicales acerca de lo que significa estar entrando y saliendo de la modernidad son las de quienes asumen las tensiones entre desterritorialización y reterritorialización. Con esto me refiero a dos procesos: la pérdida de la relación "natural" de la cultura con los territorios geográficos y sociales, y al mismo tiempo, ciertas relocalizaciones territoriales relativas, parciales, de las viejas y nuevas producciones simbólicas. (García N. , 1990)

Las comunidades tienden a reconocer también la necesidad de la convivencia, la conciliación, el asimilamiento y la aportación de legados entre culturas.

Para concluir: "Las Conferencias organizadas por la UNESCO en las décadas de los 70s y 80 s contribuyeron a sentar las bases para identificar el valor de la cultura como componente estratégico para el logro de un desarrollo integral que se manifiesta en la declaración del Decenio Mundial para la Cultura y el Desarrollo (1988 - 1997)”. (Mejía, 2007); la gestión del patrimonio como tal se presenta como una política pública importante para la permanencia de los Estados y todos los pueblos y nacionalidades que conviven dentro de ellos. 


\section{Museos populares y/o comunitarios}

El concepto de comunidad está fuertemente enraizado en las sociedades de matiz precolombino a lo largo del continente americano; elemento bajo el cual se agrupa una gran variedad de entendimientos que generan comportamientos de interdependencia humana en la que intervienen ciclos de interacción dentro de campos sociológicos y antropológicos que confluyen en legados culturales, considerados como patrimonios.

Hablar de museos populares implica el entendimiento de lo popular, pues se lo suele percibir como masivo o como algo folclorizado, a decir de García (1987) en relación al poco uso que se le daba por la década de los 70 , hasta que fue adquiriendo cierta usabilidad, "cuando sólo era utilizada por los folcloristas parecía fácil entender a qué se referían: las costumbres eran populares por su tradicionalidad, la literatura porque era oral, las artesanías porque se hacían manualmente. Tradicional, oral y manual: lo popular era el otro nombre de lo primitivo, el que se empleaba en las sociedades modernas". Cabe hasta cierto punto la definición etimológica de la palabra al significar aquello relativo al pueblo.

Para el caso de los museos, un acercamiento a lo popular, desde la perspectiva detallada de García, se tiene en el término de 'comunitario'; así, la propuesta de encajar lo popular con lo comunitario conlleva la coherencia de caracterizar a estos espacios museísticos como territorios en donde se ubica la participación de la comunidad o de sociedades comunitarias y que a la vez el discurso curatorial está en función de aquella forma "primitiva" que denota una tecnología no industrializada que forma parte de los componentes creativos al momento de establecer los objetos de exhibición o la temática intangible que pueda evocar la museografía de un museo popular.

El museo popular por lo tanto establece dos parámetros en su accionar para corresponderse con lo comunitario, el origen de sus objetos de exhibición o muestra y el discurso intencionado de base comunitaria que permite visibilizar y a la vez explorar una sociedad originaria, de ahí que en cuanto al involucramiento "de los sectores populares permitirá la apropiación y resignificación del orden dominante, mientras que para la elite (...) demarcará fundamentalmente la distinción social y la resignificación simbólica de las mercancías" (Borrega, 2006); para este argumento se distingue un matiz ideológico social en el accionar de este tipo de espacio museístico, ante lo cual lo popular "no puede identificarse por una serie de rasgos internos o un repertorio de contenidos tradicionales, 
premasivos. (...). Lo popular no se definiría por su origen o sus tradiciones, sino por su posición, la que construye frente a lo hegemónico" García (1987). Aquel posicionamiento, el del pueblo y el de mostrar las herencias y legados de los ancestros corresponde a una lectura en la cual se evoca la tradición, la costumbre y el pensamiento de lo comunitario.

Para González (2002) "el museo comunitario muestra el proceso de representación semiótico-visual de la identidad, materializado en el texto museográfico, haciendo uso del patrimonio cultural del grupo social que lo genera", ubicando una introspección social que involucra significaciones que son interpretadas desde la propia comunidad y proyectadas como lenguaje. La codificación de estos textos para un público ávido de conocer e interpretar a la cultura que se muestra, corresponde a un análisis interdisciplinario en donde se busca que el discurso patrimonial construido en estos museos sea de entendimiento práctico y de asimilación plural. Esto conlleva a establecer un claro accionar de función social, del espacio museístico, como sitio de interacción sociocultural y natural comprometiendo a las "propias comunidades en la recopilación y sistematización de los conocimientos tradicionales y de su realidad sociohistórica con una sustentación teórico metodológica para difundirlo dentro y fuera de ellas legitimando así su cultura e identidad" (Vázquez, 2008).

En definitiva, un museo popular es un espacio museístico estructurado con insumos museológicos generados desde la comunidad y que se ha construido con objetos, herramientas y pensamientos que denotan una museografía que se identifica con su entorno social, conservando su relación directa de reflejarse como un elemento más del pueblo o pueblos que representa.

\section{CONCLUSIONES}

a) Un modelo de educación patrimonial debe generar una postura objetiva en relación al discurso curatorial que se impregne dentro de un espacio museístico, evitando una parcialización en el manejo ideológico dentro de las administraciones de estos sitios y en lo que se "muestra".

b) El perfil evolutivo y re-creativo de la cultura comprende un capital de conocimiento de constante construcción, que motiva la gestión de espacios de memoria social. Una manera efectiva de darle vida y continuidad a estos sitios, es a través de un complejo informacional pedagógico de carácter patrimonial que asegure el entendimiento y 
fijación en el pensamiento de la comunidad local de aquello que surge de sí mismos y que es de su propiedad, para luego sistematizarlo en un discurso (visual, textual, etc.) que permita mostrarlo o "exhibirlo" a usuarios externos.

c) Aunque no existe una normativa especializada como política pública desde la cual se pueda aplicar una correcta ejecución de procesos que determinen que la educación tenga una rama especializada en patrimonios; se pueden desarrollar procesos metodológicos que pueden servir de base para su adaptación a diferentes contextos y condiciones del entorno, tanto social, como económico, con el fin de aplicarlos en todos los niveles sociales susceptibles de administrar patrimonios.

d) Un modelo general y dinámico de educación patrimonial debe incluir aportes desde la comunicación, de tal manera que, este enfoque transdisciplinar permita una participación integral en procesos que estén presentes de forma permanente durante la experiencia museística, tanto para los usuarios como para los administradores de estos espacios.

e) La educación patrimonial, fortalece la administración de los legados sociales, vigorizando circuitos de empleabilidad profesional, al integrar disciplinas que avivan el accionar cultural y a la vez, actúan como entes multiplicadores de conocimientos, comportamientos y prácticas, las mismas que permiten una evolución de las culturas, generando nuevos procesos, que a la vez producen dinámicas renovadas, tendientes a considerarse o determinarse como patrimonios.

f) La presente propuesta profundiza la educación patrimonial aplicada a espacios museísticos; haciendo visibilizar el componente contextual que define a cada sociedad responsable de sus propios cambios en determinados lapsos de su desarrollo vital, desde líneas discursivas con alto contenido didáctico, que permite generar un aprendizaje permanente en los individuos que viven la experiencia museística desde lo comunitario.

\section{BIBLIOGRAFÍA}

AIP. (22 de noviembre de 2020). Asociación para la Interpretación del Patrimonio. Obtenido de https://interpretaciondelpatrimonio.com/es/la-aip

AMaGA Victoria. (Septiembre de 2020). Accessible Social Media for Museums and Galleries. Obtenido de https://amagavic.org.au/assets/resources/undefined/accessible-social-media.pdf 
Amengual, I. (2015). A ras de suelo. La educación en en museos como encrucijada de discursos, pedagogías, experiencias compartidas y mucho más. Gijón: Trea.

Arias, L. (2015). Nuevos planteamientos museográficos en los museos de arte contemporáneo. Complutum, 133-143.

Asensi, A. (2016). Nuevas perspectivas de la contratación online de servicios turísticos. International Journal of Scientific Management and Tourism, 287-302.

Belloch, C. (2012). Tecnologías para el almacenamiento, recuperación, proceso y comunicación de la información. Material Docente, 1-9.

Brenholt, I. (2014). El marketing y la comunicación en el museo. En C. Padró, Mediación museística (págs. 26-27). Sintra: Mapa das Ideias.

Canal Museal. (2 de diciembre de 2020). Canal Museal. Obtenido de https://canalmuseal.com/nosotros/manifiesto-museal/

Carrión, A. (2015). Plan Nacional de Educación y Patrimonio. Madrid: IPCE.

Cerón. (2012). Museología, curaduría, gestión y muesografía. Bogotá: Ministerio de Cultura.

Cerón. (2012). Museología, curaduría, gestión y museografía. Bogotá: Ministerio de Cultura.

Claes, F. (2014). Museos Sociales. Perfiles museísticos en twitter y facebook. El profesional de la información, 594-602.

Costa, M. (2014). La museografía interactiva. Mundo científico, 48-53.

De Carli, G. (2018). El Patrimonio. Su definición, gestión y uso social. Heredia: ILAM.

De Sousa, L. (2018). Pesquisa com análise qualitativa de dados: conhecendo a Análise Temática. Arquivos Brasileiros de Psicologia, 51-67.

Delgado, S. (2020). Los retos en investigación, comunicación y educación de los museos de Lima en el marco de la COVID-19. Desde el Sur, 285-306.

Desvallés, A. (2010). Conceptos Claves de Museología. París: Colin.

Diccionario de la Lengua Española. (18 de Mayo de 2020). DLE RAE. Obtenido de https://dle.rae.es/patrimonio

Domínguez, A. (2014). Formación y educación en museos: un diálogo a varias voces. Museos.es, 97-117. 
Espacio Visual Europa. (12 de Junio de 2017). Breve Historia de la Educación en los Museos. Obtenido de https://evemuseografia.com/2015/10/22/breve-historia-de-laeducacion-en-los-museos/

Espinoza, A. (2014). Manual de accesibilidad e inclusión en museos y lugares del patrimonio cultural y natural. Gijón: Trea.

Fontal, O. (2016). Educación patrimonial: retrospectiva y prospectivas para la próxima década. Estudios pedagógicos, 415-436.

Galvis, E. (2014). Evaluación de la gestión del conocimiento: una revisión sistemática de literatura. Tendencias, 151-170.

García, I. (2015). La museografía creativa. El coleccionismo. ¿Materia o método de creación contemporánea?, 84-100.

García, I. (2020). La nueva museografía. Revista de Museología, 21-33.

Gnecco, C. (2017). Crítica de la razón arqueológica. Bogotá: Instituto Colombiano de Antropología e Historia.

González, J. (2014). Modelo de desarrollo de gestión de conocimiento en grupos de investigación. Bogotá: UPTC.

Google Arts \& Culture. (16 de noviembre de 2020). Google Arts \& Culture. Obtenido de https://artsandculture.google.com/

Guitérrez, R. (2013). Democratizar los museos. En D. Pizarro, VI Congreso Educación, Museos y Patrimonio (págs. 162-173). Santiago: OGRAMA.

Hueso, A. (2012). Metodología y técnicas cuantitativas de investigación. Valencia: Universitat Politécnica de Valencia.

ICOM. (17 de noviembre de 2020). ICOM. Obtenido de Consejo Internacional de Museos: https://icom.museum/es/news/informe-seguimiento-museos-covid-19/

Iglesias, B. (2 de enero de 2014). Mito Revista Cultural. Obtenido de http://revistamito.com/georges-henri-riviere-y-la-nueva-museologia/

ILAM. (2017). Los Diversos Patrimonios. Heredia: ILAM.

Johnson, A. (2017). The museum educator's manual: educators share successful techniques. Lanham: Rowman \& Littlefield.

l'Europe, C. d. (26 de 05 de 2019). Nouvelle stratégie et Plan d'action du Conseil de l'Europe pour la cohésion sociale. Obtenido de Council of Europe: 
https://www.coe.int/t/dg3/socialpolicies/socialcohesiondev/source/2010Strategie_ PlanAction_CohesionSociale.pdf

Ley Orgánica 3/2018 de 5 de diciembre. (2018). De protección de datos personales y de garantía de derechos digitales. BOE n 294 de 6 diciembre de 2018. Jefatura del Estado: Madrid.

Ley Orgánica de Cultura del Ecuador, de 29 de diciembre de 2016, Registro Oficial 913. (2016). Asamblea Nacional del Ecuador.

López. (2018). Aproximación a la medición de revenue management en destinos turísticos. International Journal of World of Tourism, 26-55.

López, E. (2015). Interpretación y mediación en museos. Museos.es, 41-53.

López, F. (2015). Usos del Patrimonio. Guanajuato: INAH.

Marco, B. (2015). Grado de aglomeración de los distritos turísticos españoles. EI, 21-35.

Martín, C. (2012). El prodigio de los centros de interpretación. Her\&Mus 9, 64-70.

Marulanda, C. (2013). Evaluación de la Gestión del Conocimiento en las Organizaciones . Información tecnológica, 105-116.

Monti, F. (2013). Museums and silent objects: designing effective exhibitions. Farnham: Ashgate.

Moreno, A. (2016). La mediación artística. Madrid: Octaedro.

Muñoz, C. (2012). Museología, curaduría, gestión y museografía. Bogotá: Ministerio de Cultura.

Museums Association. (Enero de 2021). Museums Change Lives. Obtenido de https://maproduction.ams3.digitaloceanspaces.com/app/uploads/2020/06/18145451/280320

17-museums-change-lives-11.pdf

Museums Association. (Enero de 2021). Power and privilege in the 21 st century museum. Obtenido de

https://maproduction.ams3.digitaloceanspaces.com/app/uploads/2020/06/18145319/Powerand-privilege-2.pdf

Pellini, J. (2017). Arqueología comercial. Madrid: Service Point.

Pizarro, D. (2013). Calidad, equidad e inclusión: El aporte desde la educación no formal. Santiago: CECA-ICOM.

Pye, E. (2016). The power of touch: handling objects in museum and heritage contexts. Abingdon: Routledge. 
Robles, P. (14 de Febrero de 2015). La validación por juicio de expertos. Obtenido de Revista Nebrija: https://www.nebrija.com/revista-linguistica/la-validacion-porjuicio-de-expertos-dos-investigaciones-cualitativas-en-linguistica-aplicada.html

Román, I. (2019). El turismo y el bienestar. Journal of Tourism and Heritage Research, 103-122.

Salazar, M. (8 de Noviembre de 2020). Correo del Maestro. Obtenido de https://www.correodelmaestro.com/publico/html5032015/capitulo5/La_educacion _patrimonial.html

Salgado, M. (2013). Diseñando un museo abierto. Buenos Aires: Wolkowicz.

Sandell, R. (2012). Museums, equality, and social justice. Abingdon: Routledge.

Santamarina, B. (2013). Los mapas geopolíticos de la Unesco. Revista de Antropología Social, 263-286.

Serrell, B. (2015). Exhibit labels: an interpretive approach. Lamham: Rawman \& Littlechild.

Soto y otros. (2013). Pensar la institución museística en términos de institución educativa y cultural. Revista Latinoamericana de Ciencias Sociales, Niñez y Juventud, 819833.

Toussaint, F. (2012). Crítica de la información de masas. Barcelona: Trillas.

UNESCO. (2003). UNESCO. Recuperado el 16 de noviembre de 2020, de https://site.inpc.gob.ec/pdfs/lotaip2020/Convencion\%202003.pdf

UNESCO. (2014). Gestión del Patrimonio Mundial Cultural. París: UNESCO.

UNESCO. (26 de Mayo de 2014). Indicadores UNESCO de Cultura para el Desarrollo. París: UNESCO. Obtenido de Patrimonio: https://es.unesco.org/creativity/sites/creativity/files/digitallibrary/cdis/Patrimonio.pdf

UNESCO. (2015). Igualdad de Género. París: UNESCO.

UNESCO. (17 de Noviembre de 2015). UNESCO. Obtenido de UNESDOC: https://unesdoc.unesco.org/ark:/48223/pf0000245176.page=14

UNESCO. (23 de junio de 2020). UNESCO CHILE. Obtenido de https://es.unesco.org/fieldoffice/santiago/cultura/patrimonio 
van Veldhuizen, A. (2017). Education Toolkit. Obtenido de http://network.icom.museum/fileadmin/user_upload/minisites/ceca/Annual_Confe rence_Docs/2017-10-08_Education_toolkit_-_e-book_EN.pdf

Vargas, S. (2017). Museografías de la nación. Historia y Grafía, Universidad Iberoamericana, 213-244.

Zavala, L. (2014). Antimanual del museólogo. México: UNAM.

Zepeda, N. (13 de noviembre de 2020). NodoCultura. Obtenido de https://nodocultura.com/2015/07/21/mediacion-como-vinculo-y-conflicto-en-elmuseo/ 